\title{
Rock and soil cutting slopes stability condition identification based on soft computing algorithms
}

\section{J. Tinoco}

ISISE - Institute for Sustainability and Innovation in Structural Engineering/ALGORITMI Research Center

School of Engineering, University of Minho, Guimares, Portugal

A. Gomes Correia

ISISE - Institute for Sustainability and Innovation in Structural Engineering

School of Engineering, University of Minho, Guimares, Portugal

P. Cortez

ALGORITMI Research Center/Department of Information Systems

School of Engineering, University of Minho, Guimares, Portugal

D. Toll

School of Engineering and Computing Sciences

University of Durham, Durham, UK.

\begin{abstract}
This study aims to develop a tool able to help decision makers to find the best strategy for slopes management tasks. It is known that one of the main challenges nowadays for every developed or countries undergoing development is to keep operational under all conditions their transportation infrastructures. However, considering the network extension and increased budget constraints such challenge is even more difficult to accomplish. In the framework of transportations networks, particularly for railway, slopes are perhaps the element for which their failure can have a strongest impact at several levels. Therefore, it is important to develop tools able to help minimizing this situation. Aiming to achieve this goal, we take advantage of the high flexible learning capabilities of Artificial Neural Networks (ANNs) and Support Vector Machines (SVMs), which have been used in the past to model complex nonlinear mappings. Both data mining algorithms were applied in the development of a classification tool able to identify the stability condition of a rock and soil cutting slopes, keeping in mind the use of information usually collected during routine inspections activities (visual information) to feed them. For that, two different strategies were followed: nominal classification and regression. Moreover, to overcome the problem of imbalanced data, three training sampling approaches were explored: no resampling, SMOTE (Synthetic Minority Over-sampling Technique) and Oversampling. The achieved results are presented and discussed, comparing the performance of both algorithms (ANN and SVM) according to each modelling strategy as well as the effect of the sampling approaches. Also, a comparison between both types of slopes is presented and discussed. An input-sensitivity analysis was applied allowing to measure the relative influence of each model attribute.
\end{abstract}

\section{INTRODUCTION}

In the framework of a transportation network, one of the biggest challenges today is to keep it operational under all conditions, mainly if we take into account its extension and the increased budget limitation for maintenance and repair tasks. Indeed, this is one of the main concerns of every developed or countries undergoing development that have invested and keep investing to build a safe and functional transportation network. Thus, taken into account the key importance of the transportation system in modern societies, it is fundamental to develop new tools able to help in its management.

In the framework of transportations networks, in particular for a railway, slopes are perhaps the element for which their failure can have the strongest impact at several levels. Therefore, it is important to develop ways to identify potential problems before they result in failures. Over time, several efforts 
have been made toward the development of a system to detect slope failures. However, most of the systems were developed for natural slopes, presenting some constraints when applied to engineered (humanmade) slopes. In addition, they have limited applicability as most of them were developed based on particular case studies or using small databases. Furthermore, another aspect that can limit its applicability is related with the information required to feed them, such as data taken from complex tests or from expensive monitoring systems. Pourkhosravani \& Kalantari (2011) summarized in their work some of the current methods for slope failure detection, which were grouped into Limit Equilibrium (LE) methods, Numerical Analysis methods, Artificial Neural Networks and Limit Analysis methods. There are also approaches based on finite elements methods (Suchomel et al. 2010), reliability analysis (Husein Malkawi, Hassan, \& Abdulla 2000), as well as some methods making use of data mining (DM) algorithms (Cheng \& Hoang 2014, Ahangar-Asr, Faramarzi, \& Javadi 2010, Yao, Tham, \& Dai 2008). More recently, a new flexible statistical system was proposed by Pinheiro, Sanches, Miranda, Neves, Tinoco, Ferreira, \& Gomes Correia (2015), based on the assessment of different factors that affect the behaviour of a given slope. By weighting the different factors, a final indicator of the slope stability condition is calculated.

In summary, most of the approaches so far proposed share the main limitations, which are related with its applicability domain or dependency on information that is difficult to obtain. In fact, the assessment of the stability condition of given slope is a multi-variable problem characterized by a high dimensionality.

Artificial Neural Networks (ANNs) and Support Vector Machines (SVMs) are two of the most well known Data Mining (DM) algorithms, which have been applied with success in different knowledge domains, such as web search, spam filters, recommender systems, and fraud detection (Domingos 2012). Also in civil engineering field, several application can be found. For example, artificial neural networks and support vector machines were applied in the study of physical and mechanical properties of jet grouting columns (Tinoco, Gomes Correia, \& Cortez 2014, Tinoco, Gomes Correia, \& Cortez 2016). Indeed, the high learning capabilities of these algorithms give them the ability to model complex nonlinear mappings. Thus, in this work we take advantage of ANNs and SVMs capabilities and fit them to a large database of rock and soil cutting slopes in order to predict the stability condition of a given slope according to a pre-defined classification scale based on four levels (classes). One of the underlying premises of this work is to identify the real stability condition of a given slope based on information that can be in a someway easily obtained during visual routine inspections. For that, more than fifty variables related with data col- lected during routine inspections as well as geometric, geological and geographic data were used to feed the models. This type of visual information is sufficient from the point of view of the network management, allowing the identification of critical zones for which more detailed information can then be obtained in order to perform more detailed stability analysis, which is out of the scope of this study. In summary, our proposal will allow to identify the stability condition level of a given rock or soil cutting slope based on visual information that, in most of the cases, can be easily obtained during routine inspections. Such novel approach is intended to support railway network management companies to allocate the available funds in the priority assets according to its stability condition.

\section{METHODOLOGY}

\subsection{Data characterization}

To fit the proposed models for stability condition identification, from this point referred to as EHC (Earthwork Hazard Category (Power, Mian, Spink, Abbott, \& Edwards 2016)), of rock and soil cutting slopes two database were used respectively. Hence, two databases were compiled containing information collected during routine inspections and complemented with geometric, geological and geographic data of each slope. Both databases were gathered by Network Rail workers and are concerned with the railway network of the UK. For each slope a class of the EHC system was defined by the Network Rail Engineers based on their experience/algorithm (Power, Mian, Spink, Abbott, \& Edwards 2016), which will be assumed as a proxy for the real stability condition of the slope for year 2015. The EHC system comprises 4 classes ("A", "B", "C" and "D") where "A" represents a good stability condition and " $D$ " a bad stability condition. In other words, the expected probability of failure is higher for class D and lower for class " $\mathrm{A}$ ".

Both databases contain a significant number of records. The rock slopes database comprises 5945 records, while the soil cutting slopes database is bigger, having 10928 records available. Figure 1 depicts the distribution of EHC classes for each database. From this analysis, it is possible to observe a high asymmetric distribution (imbalanced data), in particular for the rock cutting slopes database. In fact, more than $86 \%$ of the rock slopes are classified as " $\mathrm{A}$ " Although this type of asymmetric distribution, where most of the slopes present a low probability of failure (class " $A$ "), is normal and desirable from the safety point of view and slope network management, it can represent an important challenge for data-driven models learning, as detailed in next section. The proposed models for EHC identification of rock and soil cutting slopes were fed with more than fifty variables normally collected during routine inspections and complemented with geometric, geographic and geological 
Soil Cutting Slopes

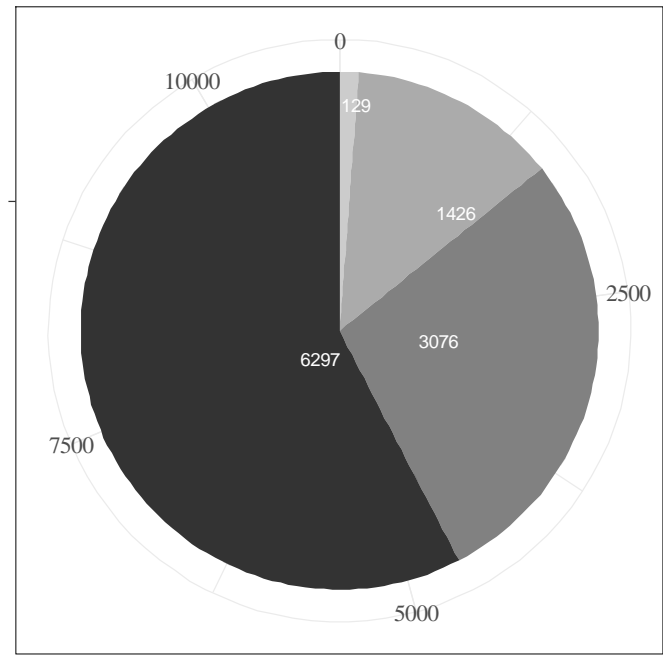

Rock Cutting Slopes

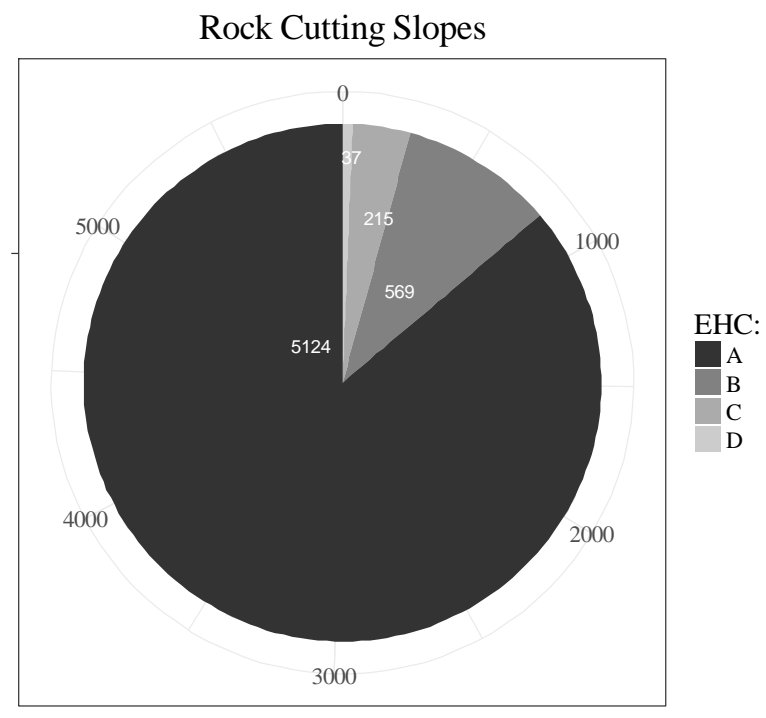

(a)

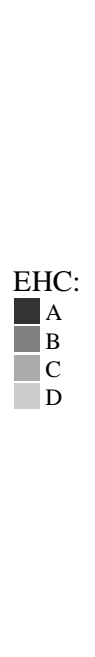

(a)

Figure 1: Rock and soil cutting slopes data distribution by EHC classes: (a) Soil cutting slopes; (b) Rock cutting slopes.

information. To be precise, 65 variables were used in the rock slopes study and 51 variables in soil cutting slopes. Since the number of analysed variables is high $(65 / 51)$, just a few examples of the variables used to feed the models are enumerated: height, slope angle, presence of rock outcrops, animal activity, presence of boulders, ground cover, rock type, dangerous trees, number of root balls, rock strength, etc.

\subsection{Modelling}

In this work we applied two of the most well known DM algorithms, namely ANNs and SVMs to model EHC prediction of rock and soil cutting slopes. These two algorithms are not new, but are supported in a strong background. Indeed, they have been applied in the past with high success in different knowledge domains including in civil engineering (Chou, Yang, \& Lin 2016, Gomes Correia, Cortez, Tinoco, \& Marques 2013). There are also some examples of ANNs and SVMs applications in slope stability analysis (Wang, Xu, \& Xu 2005, Cheng, Roy, \& Chen 2012).

ANN are learning machines that were initially inspired in functioning of the human brain (Kenig, BenDavid, Omer, \& Sadeh 2001). The information is processed using iteration among several neurons. This technique is capable of modelling complex non-linear mappings and is robust in exploration of data with noise. In this study we adopt the multilayer perceptron that contains only feedforward connections, with one hidden layer containing $H$ processing units. Because the network's performance is sensitive to $H$ (a trade-off between fitting accuracy and generalisation capability), we adopt a grid search of $\{0,2,4,6,8\}$ under an internal (i.e. applied over training data) three fold cross validation during the learning phase to find the best $H$ value. Under this grid search, the $H$ value that produced the lowest MAE (Mean Absolute Error) was selected, and then the ANN was retrained with all of the training data. The neural function of the hidden nodes was set to the popular logistic function $1 /\left(1+e^{-x}\right)$.

SVMs were initially proposed for classification tasks (Cortes \& Vapnik 1995). Then it became possible to apply SVMs to regression tasks after the introduction of the $\epsilon$-insensitive loss function (Smola $\&$ Schölkopf 2004). The main purpose of the SVM is to transform input data into a high-dimensional feature space using non-linear mapping. The SVM then finds the best linear separating hyperplane, related to a set of support vector points, in the feature space. This transformation depends on a kernel function. In this work the popular Gaussian kernel was adopted. In this context, its performance is affected by three parameters: $\gamma$, the parameter of the kernel; $\mathrm{C}$, a penalty parameter; and $\epsilon$ (only for regression), the width of an $\epsilon$-insensitive zone (Safarzadegan Gilan, Bahrami Jovein, \& Ramezanianpour 2012). The heuristics proposed by (Cherkassky \& Ma 2004) were used to define the first two parameter values, $\mathrm{C}=3$ (for a standardised output) and $\epsilon=\hat{\sigma} / \sqrt{N}$, where $\hat{\sigma}=1.5 / N \cdot \sum_{i=1}^{N}\left(y_{i}-\hat{y}_{i}\right)^{2}, y_{i}$ is the measured value, $\hat{y}_{i}$ is the value predicted by a 3-nearest neighbour algorithm and $N$ is the number of examples. A grid search of $2^{\{-1,-3,-7,-9\}}$ was adopted to optimise the kernel parameter $\gamma$, under the same internal threefold cross-validation scheme adopted for ANN.

As a first attempt, EHC prediction of rock and soil cutting slopes was approached following a nominal classification strategy. Then, aiming to improve the models performance, the problem was also addressed following a regression strategy, adopting a regression scale where $\mathrm{A}=1, \mathrm{~B}=2, \mathrm{C}=4, \mathrm{D}=10$, which was 
that leading to the best performance.

In addition, in order to minimize the effect of the imbalanced data (see Figure 1), Oversampling (Ling \& Li 1998) and SMOTE (Synthetic Minority Oversampling Technique) (Chawla, Bowyer, Hall, \& Kegelmeyer 2002) approaches were applied over the training data before fitting the models. When approaching imbalanced classification tasks, where there is at least one target class label with a smaller number of training samples when compared with other target class labels, the simple use of a soft computing training algorithm will lead to data-driven models with better prediction accuracies for the majority classes and worst classification accuracies for the minority classes. Thus, techniques that adjust the training data in order to balance the output class labels, such as Oversampling and SMOTE, are commonly used with imbalanced datasets. In particular, Oversampling is a simple technique that randomly adds samples (with repetition) of the minority classes to the training data, such that the final training set is balanced. SMOTE is a more sophisticated technique that creates "new data" by looking at nearest neighbours to establish a neighbourhood and then sampling from within that neighbourhood. It operates on the assumptions that the original data is similar because of proximity. More recently, Torgo, Branco, Ribeiro, \& Pfahringer (2015) adapted the SMOTE method for regression tasks. We note that the different sampling approaches were applied only to training data, used to fit the data-driven models, and the test data (as provided by the 5-fold procedure) was kept without any change.

For models evaluation and comparison, we calculated three classification metrics: recall, precision and $F_{1-\text { score }}$ (Hastie, Tibshirani, \& Friedman 2009). The recall measures the ratio of how many cases of a certain class were properly captured by the model. In other words, the recall of a certain class is given by TruePositives/(TruePositives + FalseNegatives). On the other hand, the precision measures the correctness of the model when it predicts a certain class. More specifically, the precision of a certain class is given by TruePositives/(TruePositives + FalsePositives). The $\mathrm{F}_{1 \text {-score }}$ was also calculated, which represent a trade-off between the recall and precision of a class. The $F_{1-\text { score }}$ correspond to the harmonic mean of precision and recall, according to the following expression:

$$
F_{1-\text { score }} s=2 \cdot \frac{\text { precision } \cdot \text { recall }}{\text { precision }+ \text { recall }}
$$

For all three metrics, the higher the value, the better are the predictions, ranging from $0 \%$ to $100 \%$.

The generalization capacity of the models was accessed through a 5-fold cross-validation approach under 20 runs (Hastie, Tibshirani, \& Friedman 2009). This means that each modelling setup is trained $5 \times$
$20=100$ times. Also, the three prediction metrics are always computed on test unseen data (as provided by the 5-fold validation procedure).

All experiments were conducted under the R statistical environment (Team 2009). ANN and SVM algorithms were trained using the rminer package (Cortez 2010), which facilitates its implementation, as well as different validation approaches such as the crossvalidation adopted in this work.

\section{RESULTS AND DISCUSSION}

Following are presented and discussed the achieved performance in EHC prediction of rock and soil cutting slopes through the application of soft computing techniques, comparing both soft computing algorithms (ANN and SVM) performance for each one of the two implemented strategies (nominal classification and regression) as well as for the three training sampling approaches explored: Normal (no resampling), OVERed (Oversampling) and SMOTEd (SMOTE). A particular emphases is also given to the comparison between soil and rock cutting slopes studies.

Tables 1 and 2 give an overview of all models performance in soil and rock cutting slopes respectively, based on recall, precision and $\mathrm{F}_{1 \text {-score }}$.

Concerning to soil cutting slopes study, a very promising performance is observed, namely according to a nominal classification strategy, which learned better EHC prediction than following a regression strategy as shown in Figure 2a. For example, soil cutting slopes of class "A" can be correctly identified, particularly by ANN model, with or without resampling. Also for classes " $\mathrm{B}$ " and " $\mathrm{C}$ " a promising performance is observed, with an $\mathrm{F}_{1 \text {-score }}$ around 55\%, in particular by the ANN algorithm. Concerning the class "D", although an $\mathrm{F}_{1 \text {-score }}$ lower than $36 \%$ was achieved, the obtained value for recall metric around $57 \%$ shows a good performance for class " $\mathrm{D}$ “ prediction according to ANN algorithm. Following a regression strategy, and by comparison with the nominal classification strategy, the main differences are related with the effect of the sampling approaches, which is not so relevant, particularly for the minority classes. Moreover, analysing Figure 3a that shows the relation between observed and predicted EHC values according to the best fit, we can see that the models performance is very promising. Indeed, according to a nominal classification strategy with SMOTE resampling, ANN algorithm is able to predict correctly around $57 \%$ of soil cutting slopes of class "D", which represents a very promising performance if we take into account that this is the minority class. For class "C", although the accuracy is lower (around 40\%), when not predicted as "C" they are classified as belonging to the closest class, that is, " $\mathrm{B}$ " or " $\mathrm{D}$ ". This type of misclassification is also observed for classes "A", "B" and "D", which can be interpreted as an ad- 
vantage. Concerning to classes "A" and "B", the ANN model was able to identify it very accurately.

Relating to rock cutting slopes study, the achieved performance is somewhat lower, either following a nominal classification or regression strategies. Although a very high performance is observed in class "A" identification $\left(\mathrm{F}_{1 \text {-score }}\right.$ higher than $\left.95 \%\right)$, for class "C" and particularly for class " $\mathrm{D}$ ", all models evidence difficulties in predicting these classes correctly. In fact, and using $F_{1 \text {-score }}$ as reference, the best performance in identification of slopes of class " $\mathrm{D}$ " is lower than $14 \%$ (see Figure $2 b$ ) which was achieved by the ANN algorithm following a nominal classification strategy with SMOTE re-sampling. From Figure 3b analysis, which plots the relation between observed and predicted EHC values based on ANN algorithm following a nominal classification with oversampling (best fit), it is clear the model difficulties in correctly predicting class " $C$ " and particularly class " $D$ ", for which the expected probability of failure is higher. As shown, only around $12 \%$ of rock cutting slopes classified as "D" are correctly identified, which represents a low performance, namely when compared with soil cutting slopes study. Overall, these results show that the methodology applied for EHC prediction of rock cutting slopes needs future development in order to overcome this gap.

Comparing the achieved results of soil and rock cutting slopes studies, the proposed models for soil cutting slopes are more effective, namely in the identification of classes "C" and "D" for which the probability of failure is higher (see Figure 2). A possible explanation for the lower performance, namely in classes " $C$ " and " $D$ " identification of rock cutting slopes could be related with the EHC classes being assumed as representative of the real stability condition of each slope. Indeed, analysing the number of slope failures by EHC class for rock slopes there are some indications that the classification attributed to each rock slope could lack some accuracy as reported in the work of Power, Mian, Spink, Abbott, \& Edwards (2016), that used the same source of information. It would be expected that most of the failures would occur in slopes of classes " $C$ " and mainly " $D$ ". However, for rock slopes such behaviour is not observed as reported on Power, Mian, Spink, Abbott, \& Edwards (2016). In fact, the number of failures for each EHC class is almost constant from " $\mathrm{A}$ " to " $\mathrm{D}$ ", particularly when compared with soil cuttings. For example, the number of failures observed in rock cutting slopes of class " $C$ " is only twice higher when compared to class " $A$ ". This observation shows that the defined classes for rock slopes have a poor correlation with actual failures.

These results show that a deeper data analysis is required, particularly in the study of rock cutting slopes. For example, the number of variables taken as model attributes might be too high and may be influencing the generalization performance of the models. Aiming to check if a better generalization could be achieved using the most relevant inputs, we performed additional experimentation using a fast feature selection method that is based on a sensitive analysis (Cortez \& Embrechts 2013), which allows to measure the relative importance of each input of a classification or regression method. Taken as reference the two models that achieved the overall best performance in EHC prediction of soil and rock cutting slopes (see Figure 3), we applied the sensitivity analysis to measure the relevance of each input variable in EHC prediction. Figure 4 shows the relative importance of the 20 most relevant variables in both soil and rock cutting slopes studies. Following these results, all models were re-trained (including both strategies and the three re-sampling approaches) considering only the 12 and 16 most relevant variable in soil and rock cutting slopes studies respectively. Using $\mathrm{F}_{1 \text {-score }}$ as comparison metric, Table 3 shows the difference between the full models (with 51/65 inputs for soil and rock slopes respectively) and feature selection ones (with 12/16 most relevant inputs). The results from Table 3 show that the feature selection tends to present a lower performance, with lower $\mathrm{F}_{1 \text {-score }}$ values. Thus, in the light of the achieved results, and as a future works, we intend to apply a more sophisticated feature selection method aiming to improve models performance. For instance, by using a multi-objective evolutionary computation method that simultaneously maximizes prediction performance and minimizes the number of inputs used.

As a final observation, and considering the overall performance of all models, we would like to underline the potential of soft computing algorithms, namely the ANNs, in EHC prediction of soil and rock cutting slopes.

\section{ACKNOWLEDGEMENTS}

This work was supported by FCT - "Fundação para a Ciência e a Tecnologia", within ISISE, project UID/ECI/04029/2013 as well Project Scope: UID/CEC/00319/2013 and through the post-doctoral Grant fellowship with reference SFRH/BPD/94792/2013. This work was also partly financed by FEDER funds through the Competitivity Factors Operational Programme - COMPETE and by national funds through FCT within the scope of the project POCI-01-0145-FEDER-007633. This work has been also supported by COMPETE: POCI-01-0145-FEDER-007043. A special thanks goes to Network Rail that kindly made available the data (basic earthworks examination data and the Earthworks Hazard Condition scores) used in this work. 
Table 1: Metrics in EHC prediction of rock slopes (best values in bold)

\begin{tabular}{|c|c|c|c|c|c|c|c|c|c|c|c|c|c|c|c|}
\hline & \multirow{2}{*}{ Model } & \multirow{2}{*}{ Approach } & \multirow{2}{*}{ AUS } & \multicolumn{4}{|c|}{ Recall } & \multicolumn{4}{|c|}{ Precision } & \multicolumn{4}{|c|}{$F_{1 \text {-score }}$} \\
\hline & & & & A & B & $\mathrm{C}$ & D & A & B & $\mathrm{C}$ & D & A & B & $\mathrm{C}$ & D \\
\hline \multirow{6}{*}{ 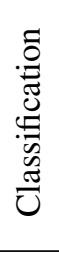 } & & Norr & 0.46 & 96.23 & 52.95 & 20.40 & 3.65 & 94.66 & 49.06 & 39.22 & 13.71 & 95.44 & 50.93 & 26.84 & 5.77 \\
\hline & ANN & & & 88.1 & 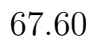 & 2 & 17.3 & 98 & & 26 & 10.89 & 1 & 48.95 & 30.49 & 13.37 \\
\hline & & OVERed & 0. & 90.21 & 67.96 & 39.58 & 12.84 & 98.01 & 41.27 & 33.47 & 12.70 & 93.95 & 51.35 & 36.27 & 12.77 \\
\hline & & Norn & 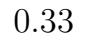 & 97.39 & 39.79 & & & 91.63 & 48,57 & 42. & 18.75 & 4.42 & 43.74 & 11.20 & \\
\hline & SVM & SMOT & 0.29 & 85.53 & 82.64 & 2.0 & 1.49 & 97.24 & 33.08 & 34.36 & 17.19 & 91.01 & 47.25 & 3.90 & 2.74 \\
\hline & & OVERed & 0.13 & 99.78 & 7.14 & 0.00 & 0.00 & 86.95 & 62.83 & $N A$ & 0.00 & 92.92 & 12.82 & $N A$ & $N A$ \\
\hline \multirow{4}{*}{ 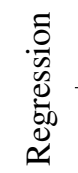 } & & $\mathrm{N}$ & 0.43 & 93 & 48.3 & 41.7 & 3.3 & 95.01 & 41.38 & 40.1 & 30.49 & 94.35 & 44.57 & 40.96 & 6. \\
\hline & & SMC & 0.35 & 85.97 & 68.37 & 45.8 & 4.32 & 98.07 & 33.85 & 32.95 & 35.56 & 91.62 & 45.28 & 38.34 & 7.70 \\
\hline & & $\mathrm{N}$ & 0.34 & 96. & 49.8 & & $0 .($ & 92 & 46. & 54 & & 94 & 48 & 0. & \\
\hline & $\mathrm{M}$ & & & 77 & 93.15 & 11 & 0.0 & 99 & 27.61 & 48. & $N$ & 86.86 & 42.59 & 18.08 & $N$ \\
\hline
\end{tabular}

Table 2: Metrics in EHC prediction of soil cutting slopes (best values in bold)

\begin{tabular}{|c|c|c|c|c|c|c|c|c|c|c|c|c|c|c|c|}
\hline & \multirow{2}{*}{ Model } & \multirow{2}{*}{ Approach } & \multirow{2}{*}{ AUS } & \multicolumn{4}{|c|}{ Recall } & \multicolumn{4}{|c|}{ Precision } & \multicolumn{4}{|c|}{$\mathrm{F}_{1 \text {-score }}$} \\
\hline & & & & A & B & $\mathrm{C}$ & $\mathrm{D}$ & A & B & $\mathrm{C}$ & $\mathrm{D}$ & A & B & $\mathrm{C}$ & $\mathrm{D}$ \\
\hline \multirow{6}{*}{ 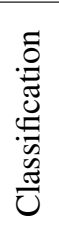 } & & Norr & 15 & 90.36 & 4 & - & 4 & & 0.36 & & r & 8.77 & 2.13 & 1.53 & \\
\hline & ANN & Ed & 0.08 & 80.87 & 66.59 & 46.07 & 56.78 & 91.68 & 54.49 & 51 & 21.63 & 85.94 & 59.94 & 48.62 & 31.33 \\
\hline & & Or & 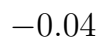 & 82.05 & 58 & 63.77 & 38.41 & 01 & 2 & 49.77 & 33 & 86.35 & 56.82 & 55.91 & 35.91 \\
\hline & & $\mathrm{N}$ & -0.12 & 90.33 & 6.82 & 3411 & 2.2 & 85 & 58.34 & 57.71 & 22.31 & 88.56 & 62.29 & 42.88 & 4.09 \\
\hline & SVM & & 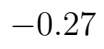 & 73.65 & 79 & 24.96 & 24.88 & 91 & 47.90 & 53 & 30 & 1 & 59 & 34.05 & 27.53 \\
\hline & & OVERed & 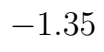 & 94.79 & 24 & 1.54 & 13 & 63 & 52.35 & 62.98 & 62.96 & 75.87 & 33.60 & 3.01 & 2.59 \\
\hline \multirow{4}{*}{ 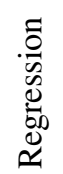 } & & Norn & -0.05 & 87.4 & 17 & 47.94 & 25.62 & 87.74 & 57.88 & 59 & & 87.57 & 61.00 & 52.98 & 32 \\
\hline & & SMOTEd & -0.03 & 85.34 & 68.68 & 48.53 & 23.64 & 89.32 & 57.00 & 60.23 & 54.08 & 87.28 & 62.30 & 53.75 & 32.90 \\
\hline & & Normal & -0.16 & 83.66 & 82.02 & 15.7 & 0.00 & 91.07 & 52.89 & 60.00 & $N A$ & 87.21 & 64.31 & 24.89 & $N A$ \\
\hline & VIMI & & & 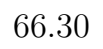 & 85.38 & 33.77 & 0.62 & T & 45.81 & 66.37 & 66.67 & 77.56 & 59.63 & 44.76 & 1.23 \\
\hline
\end{tabular}

Table 3: Difference between $\mathrm{F}_{1 \text {-score }}$ values of the full input model with a feature selection model that included the most relevant inputs according to a sensitivity analysis procedure.

\begin{tabular}{|c|c|c|c|c|c|c|c|c|c|c|}
\hline & \multirow{2}{*}{ Model } & \multirow{2}{*}{ Approach } & \multicolumn{4}{|c|}{ Soil cutting slopes } & \multicolumn{4}{|c|}{ Rock cutting slopes } \\
\hline & & & A & $\mathrm{B}$ & $\mathrm{C}$ & $\mathrm{D}$ & A & $\mathrm{B}$ & $\mathrm{C}$ & $\mathrm{D}$ \\
\hline \multirow{6}{*}{ 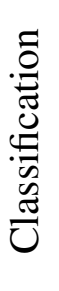 } & & Normal & 7.11 & 16.00 & 18.81 & 15.09 & 1.53 & 13.9 & 19.27 & 3.72 \\
\hline & ANN & SMOTEd & 9.15 & 12.25 & 23.60 & 19.04 & 2.38 & 7.51 & 3.15 & 5.26 \\
\hline & & OVERed & 8.82 & 20.31 & 16.31 & 21.00 & 3.28 & 12.96 & 10.31 & 6.30 \\
\hline & & Norma & 7.44 & 15.26 & 28.56 & 3.49 & 0.90 & 13.34 & 7.31 & $N A$ \\
\hline & SVM & SMOTEd & 6.23 & 13.78 & -1.12 & 13.17 & 90.87 & 29.78 & $N A$ & $N A$ \\
\hline & & OVERed & -0.49 & -17.01 & -8.51 & -2.21 & 0.91 & -25.02 & $N A$ & $N A$ \\
\hline \multirow{4}{*}{ 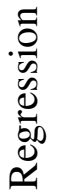 } & $\Delta \mathbf{N N}$ & Normal & 10.00 & 9.40 & 17.25 & 25.82 & 1.23 & -2.20 & 14.46 & 5.81 \\
\hline & AIVN & SMOTEd & 10.38 & 10.90 & 17.44 & 28.29 & 1.24 & 1.47 & 9.66 & $N A$ \\
\hline & $M$ & Norn & 6.49 & 14.74 & 1.69 & $N A$ & 0.74 & 3.65 & 0.18 & $N A$ \\
\hline & $S$ V IVI & SMOTEd & 0.72 & 11.79 & 17.27 & $N A$ & -1.70 & -0.56 & 2.34 & $N A$ \\
\hline
\end{tabular}


Approach: $\square$ Normal $\square$ SMOTEd OVERed

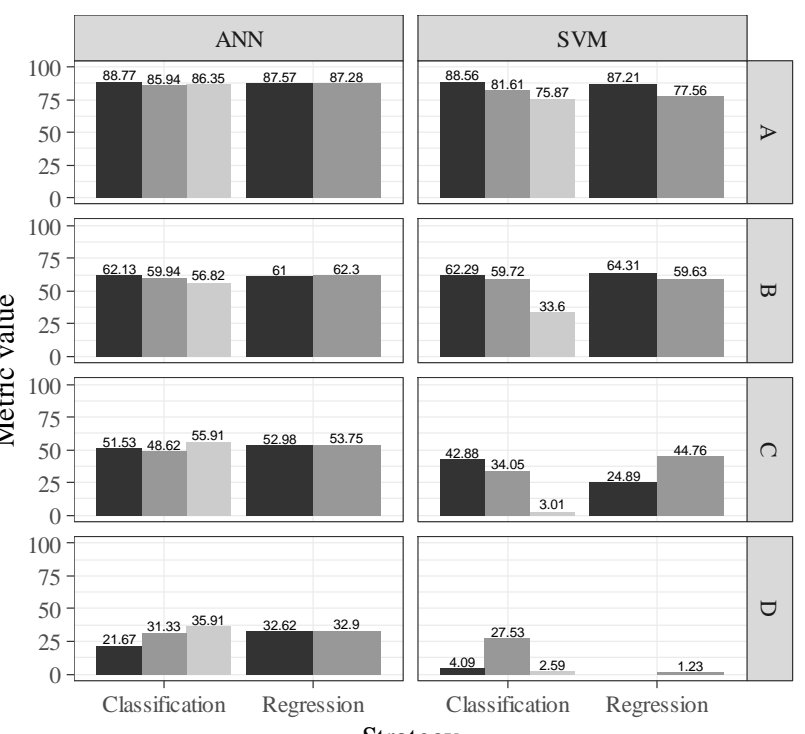

(a)

Strategy

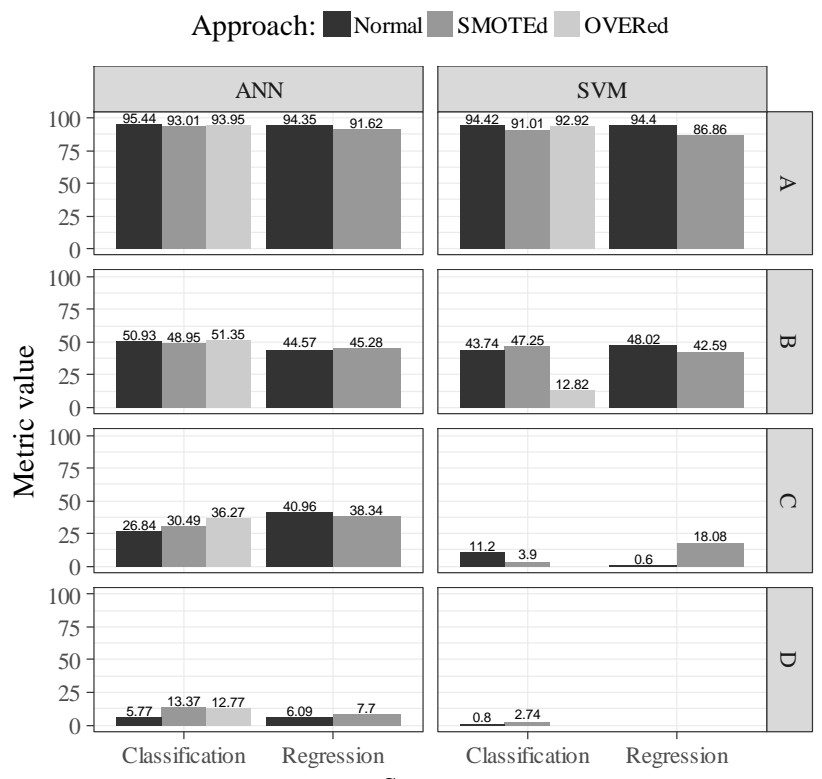

(b)

Figure 2: Nominal classification and Regression strategies performance comparison based on $\mathrm{F}_{1 \text {-score }}$ : (a) Soil cutting slopes study; (b) Rock cutting slopes study.

\section{ANN :: SMOTEd - Nominal Classification}

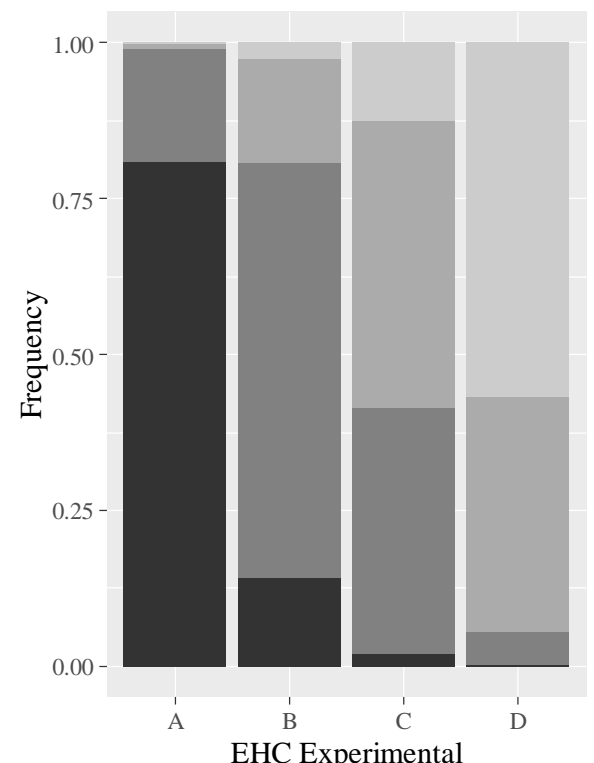

(a)

Figure 3: ANN models performance comparison according to a nominal classification strategy in EHC prediction of: (a) Soil cutting slopes following an SMOTEd approach; (b) Rock cutting following a OVERed approach.

\section{REFERENCES}

Ahangar-Asr, A., A. Faramarzi, \& A. A. Javadi (2010). A new approach for prediction of the stability of soil and rock slopes. Engineering Computations 27(7), 878893.

Chawla, N. V., K. W. Bowyer, L. O. Hall, \& W. P. Kegelmeyer (2002). Smote: synthetic minority oversampling technique. Journal of artificial intelligence research 32, 321-357.

Cheng, M.-Y. \& N.-D. Hoang (2014). Slope collapse prediction using bayesian framework with k-nearest neighbor density estimation: Case study in taiwan. Journal of Computing in Civil Engineering 30(1), 04014116.

Cheng, M.-Y., A. F. Roy, \& K.-L. Chen (2012). Evolutionary risk preference inference model using fuzzy support

\section{ANN :: OVERed - Nominal Classification}

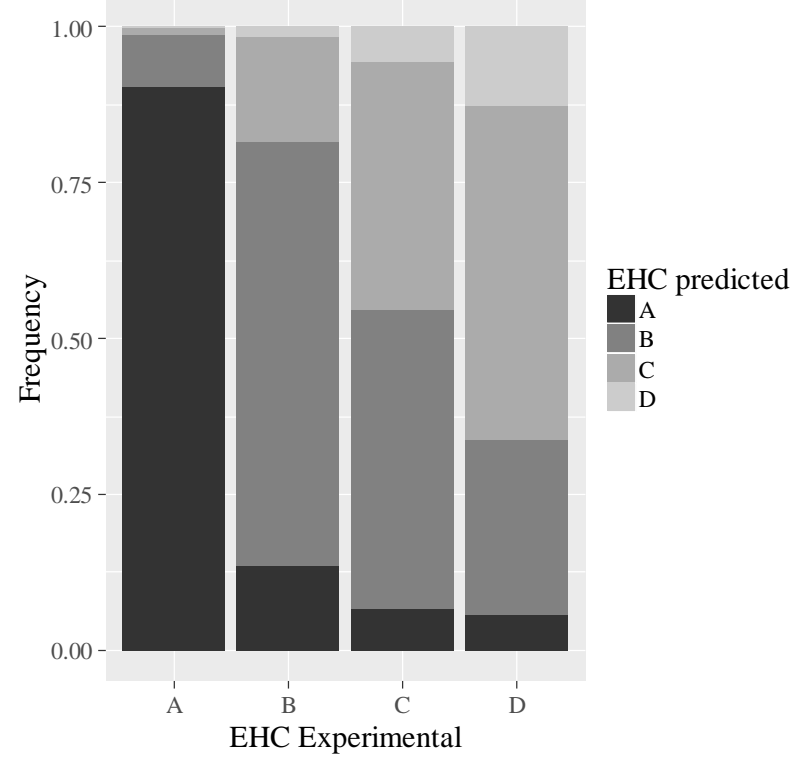




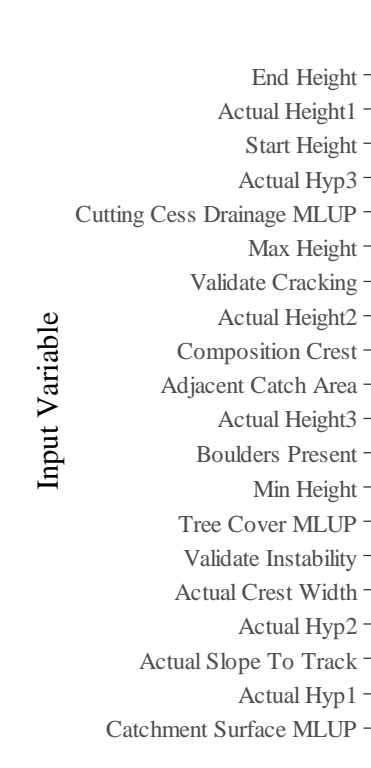

(a)
ANN :: OVERed - Nominal Classification

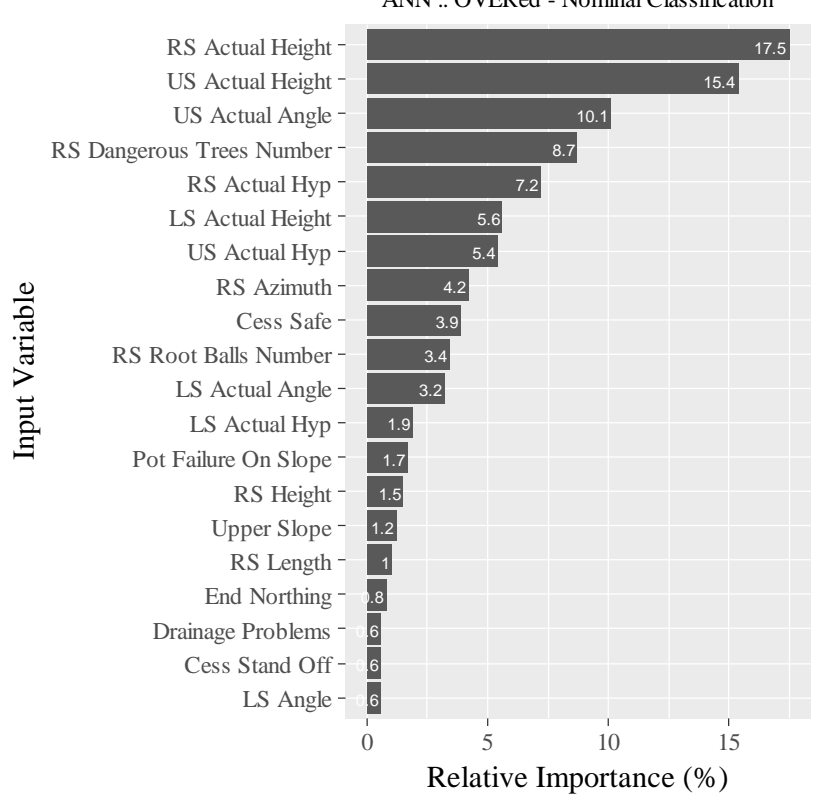

Figure 4: Relative importance bar plot of the 20 most relevant variables according to ANN models based on a nominal classification strategy in EHC prediction of: (a) Soil cutting following a SMOTEd approach; (b) Rock slopes following an OVERed approach.

\section{LNAI 6171, Springer.}

Cortez, P. \& M. Embrechts (2013). Using sensitivity analysis and visualization techniques to open black box data mining models. Information Sciences 225, 1-17.

Domingos, P. (2012). A few useful things to know about machine learning. Communications of the ACM 55(10), $78-87$.

Gomes Correia, A., P. Cortez, J. Tinoco, \& R. Marques (2013, Mai). Artificial intelligence applications in transportation geotechnics. Geotechnical and Geological Engineering 31(3), 861-879. doi:10.1007/s10706-0129585-3.

Hastie, T., R. Tibshirani, \& J. Friedman (2009). The Elements of Statistical Learning: Data Mining, Inference, and Prediction (Second Edition ed.). Springer-Verlag New York.

Husein Malkawi, A. I., W. F. Hassan, \& F. A. Abdulla (2000). Uncertainty and reliability analysis applied to slope stability. Structural Safety 22(2), 161-187.

Kenig, S., A. Ben-David, M. Omer, \& A. Sadeh (2001). Control of properties in injection molding by neural networks. Engineering Applications of Artificial Intelligence 14(6), 819-823.

Ling, C. X. \& C. Li (1998). Data mining for direct marketing: Problems and solutions. In $K D D$, Volume 98, pp. 73-79.

Pinheiro, M., S. Sanches, T. Miranda, A. Neves, J. Tinoco, A. Ferreira, \& A. Gomes Correia (2015). A new empirical system for rock slope stability analysis in exploitation stage. International Journal of Rock Mechanics and Mining Sciences 76, 182-191. http://dx.doi.org/10.1016/j.ijrmms.2015.03.015.

Pourkhosravani, A. \& B. Kalantari (2011). A review of current methods for slope stability evaluation. Electronic Journal of Geotechnical Engineering 16.

Power, C., J. Mian, T. Spink, S. Abbott, \& M. Edwards (2016). Development of an evidence-based geotechnical asset management policy for network rail, great britain. Procedia Engineering 143, 726-733.

Safarzadegan Gilan, S., H. Bahrami Jovein, \& A. Ramezanianpour (2012). Hybrid support vector regressionparticle swarm optimization for prediction of compressive strength and rcpt of concretes containing metakaolin. Construction and Building Materials 34, 321-329.

Smola, A. \& B. Schölkopf (2004). A tutorial on support vector regression. Statistics and Computing 14(3), 199222.

Suchomel, R. et al. (2010). Comparison of different probabilistic methods for predicting stability of a slope in spatially variable $c-\varphi$ soil. Computers and Geotechnics 37(1), 132-140.

Team, R. (2009). R: A language and environment for statistical computing. R Foundation for Statistical Computing, Viena, Austria. Web site: http://www.r-project.org/.

Tinoco, J., A. Gomes Correia, \& P. Cortez (2014, January). Support vector machines applied to uniaxial compressive strength prediction of jet grouting columns. Computers and Geotechnics 55, 132-140. http://dx.doi.org/10.1016/j.compgeo.2013.08.010.

Tinoco, J., A. Gomes Correia, \& P. Cortez (2016, June). Jet grouting column diameter prediction based on a datadriven approach. European Journal of Environmental and Civil Engineering O(0), 1-22.

Torgo, L., P. Branco, R. Ribeiro, \& B. Pfahringer (2015). Resampling strategies for regression. Expert Systems 32(3), 465-476.

Wang, H., W. Xu, \& R. Xu (2005). Slope stability evaluation using back propagation neural networks. Engineering Geology 80(3), 302-315.

Yao, X., L. Tham, \& F. Dai (2008). Landslide susceptibility mapping based on support vector machine: a case study on natural slopes of hong kong, china. Geomorphology 101(4), 572-582. 